\title{
Trafficking of Membrane-Associated Proteins to Cone Photoreceptor Outer Segments Requires the Chromophore 11-cis-Retinal
}

\author{
Houbin Zhang, ${ }^{1}$ Jie Fan, ${ }^{3}$ Sha Li, ${ }^{1}$ Sukanya Karan, ${ }^{5}$ Baerbel Rohrer, ${ }^{3}$ Krzysztof Palczewski, ${ }^{4}$ Jeanne M. Frederick, ${ }^{1}$ \\ Rosalie K. Crouch, ${ }^{3}$ and Wolfgang Baehr ${ }^{1,2,5}$ \\ Departments of ${ }^{1}$ Ophthalmology and ${ }^{2}$ Neurobiology and Anatomy, University of Utah Health Science Center, Salt Lake City, Utah 84132, ${ }^{3}$ Department of \\ Ophthalmology, Medical University of South Carolina, Charleston, South Carolina 29425, ${ }^{4}$ Department of Pharmacology, Case Western Reserve University, \\ Cleveland, Ohio 44106, and ${ }^{5}$ Department of Biology, University of Utah, Salt Lake City, Utah 84112
}

Lecithin retinol acyl transferase (LRAT) and retinal pigment epithelium protein 65 (RPE65) are key enzymes of the retinoid cycle. In Lrat $^{-1-}$ and Rpe65 $5^{-1-}$ mice, models of human Leber congenital amaurosis, the retinoid cycle is disrupted and 11-cis-retinal, the chromophore of visual pigments, is not produced. The $L$ rat ${ }^{-1-}$ and $R p e 65^{-1-}$ retina phenotype presents with rapid sectorial cone degeneration, and the visual pigments, S-opsin and M/L-opsin, fail to traffic to cone outer segments appropriately. In contrast, rod opsin traffics normally in mutant rods. Concomitantly, guanylate cyclase 1, cone T $\alpha$-subunit, cone phosphodiesterase 6 $\alpha^{\prime}$ (PDE6 $\alpha^{\prime}$ ), and GRK1 (G-protein-coupled receptor kinase 1; opsin kinase) are not transported to $\mathrm{Lrat}^{-1-}$ and $\mathrm{Rpe}^{-1-}$ cone outer segments. Aberrant localization of these membrane-associated proteins was evident at postnatal day 15, before the onset of ventral and central cone degeneration. Protein levels of cone T $\alpha$ and cone PDE6 $\alpha$ ' were reduced, whereas their transcript levels were unchanged, suggesting posttranslational degradation. In an Rpe65 ${ }^{-I-} R h o^{-I-}$ double knock-out model, trafficking of cone pigments and membrane-associated cone phototransduction polypeptides to the outer segments proceeded normally after 11-cis-retinal administration. These results suggest that ventral and central cone opsins must be regenerated with 11-cis-retinal to permit transport to the outer segments. Furthermore, the presence of 11-cis-retinal is essential for proper transport of several membrane-associated cone phototransduction polypeptides in these cones.

Key words: cone visual pigments; LRAT (lecithin retinol acyl transferase); RPE65; retinoid isomerase; membrane protein trafficking; Leber congenital amaurosis

\section{Introduction}

Photon absorption by photosensitive visual pigments in rod and cone photoreceptors initiates signal transduction and vision (Palczewski, 2006). Visual pigments are G-proteincoupled receptors using as their ligand 11-cis-retinal, which isomerizes to the all-trans form with light. Regeneration of

Received Jan. 23, 2008; revised March 7, 2008; accepted March 12, 2008.

This work was supported by National Institutes of Health (NIH) Grants EY04939 (R.K.C.), EY13520 (B.R.), EY09339 (K.P.), EY14793 (Medical University of South Carolina vision core), EY08123 (W.B.), EY014800-039003 (National Eye Institute core grant), by a grant from the Foundation Fighting Blindness (K.P.), a Foundation Fighting Blindness center grant to the University of Utah, and unrestricted grants to the Departments of Ophthalmology at the Medical University of South Carolina and the University of Utah from Research to Prevent Blindness (RPB) (New York, NY) R.K.C. is an RPB Senior Scientific Investigator, and B.R. is an RPB Olga Keith Weiss Scholar. The animal studies were conducted in a facility constructed with support from the Research Facilities Program and Grant C06 RR015455 from the NIH National Center for Research Resources. We thank Drs. Ching-Kang Chen (Virginia Commonwealth University, Richmond, VA) for anti-rod transducin- $\alpha$ antibody, Cheryl Craft (University of Southern California, Los Angeles, (A) for anti-mouse cone arrestin antibody, Tiansen Li (Harvard University, Cambridge, MA) for anti-cone PDE6 $\alpha$ ' antibody, Stefan Heller (Stanford University, Palo Alto, CA) for anti-R9AP antibody, and Nils Brose (Max Planck Institute for Experimental Medicine, Goettingen, Germany) for anti-complexin III antibody.

Correspondence should be addressed to Wolfgang Baehr, John A. Moran Eye Center, University of Utah Health Science Center, Salt Lake City, UT 84132. E-mail: wbaehr@hsc.utah.edu.

D0I:10.1523/JNEUROSCI.0317-08.2008

Copyright $\odot 2008$ Society for Neuroscience $\quad 0270-6474 / 08 / 284008-07 \$ 15.00 / 0$ 11-cis-retinal in the retinoid cycle is essential for maintaining normal visual function (Wald, 1968). After photobleaching, all-trans-retinal separates from rhodopsin and cone opsins and is reduced to all-trans-retinol (Maeda et al., 2007). Alltrans-retinol, in turn, is exported to the retinal pigment epithelium (RPE), where it is converted into retinyl esters by lecithin retinol acyltransferase (LRAT). All-trans retinyl esters are the substrate for RPE protein 65 (RPE65), recently identified as the retinoid isomerase that generates 11-cis-retinol (Jin et al., 2005; Moiseyev et al., 2005; Redmond et al., 2005). After oxidation to 11-cis-retinal, the chromophore is exported back to the photoreceptors for the regeneration of functional pigments.

LRAT and RPE65 knock-out mice have been shown to lack 11-cis-retinal for visual pigment regeneration. In the $\mathrm{Lrat}^{-1-}$ mouse, only trace amounts of all-trans-retinyl esters were detected, consistent with blockage of the retinoid cycle at the esterification step (Batten et al., 2004). In the Rpe65 ${ }^{-/-}$mouse, retinyl esters accumulate, and 11-cis-retinal cannot be produced (Redmond et al., 1998b). Administration of exogenous 9- or 11-cisretinal can significantly improve rod function in $R p e 65^{-/-}$mice, even as old as 18 months (Van Hooser et al., 2002; Rohrer et al., 
2003). Cones and rods are supported by the retinoid cycle in a similar, but not identical, manner. In primates, RPE65 and its substrate all-trans-retinyl esters are localized at highest concentrations in the RPE cells that cover the foveal region of the retina (Jacobson et al., 2007). The possibility has been raised that additional processes may exist that generate 11-cis-retinal for cones supplementing the major RPE65-dependent pathway (Mata et al., 2005; Muniz et al., 2007; Schonthaler et al., 2007). Residual function of RPE65-Leber congenital amaurosis (LCA) cones and prolonged cone photoreceptor survival in human patients support this speculation (Jacobson et al., 2007).

Unlike rods, cone photoreceptors degenerate rapidly (Znoiko et al., 2005), and notably, cone opsins (both S-opsin and M/Lopsin) (Ebrey and Koutalos, 2001) were found to mislocalize in the Rpe $65^{-1-}$ cones and unable to traffic to the cone outer segments (COSs) properly (Rohrer et al., 2005). Administration of 11-cis-retinal before onset of degeneration [postnatal day 10 (P10)] prevented cone opsin mistrafficking and slowed the rate of degeneration (Rohrer et al., 2005). Here, we explored the consequences of 11-cis-retinal absence in Rpe65 $5^{-1-}$ and $\mathrm{Lrat}^{-1-}$ mice on trafficking of integral and peripheral membrane-associated proteins (PMPs) to cone outer segments. We show that cone opsins mislocalized to the inner segment and synaptic pedicle at $\mathrm{P} 15$, and polypeptides of the cone phototransduction cascade [including guanylate cyclase 1 (GC1) and G-protein-coupled receptor kinase 1 (GRK1)] failed to transport to the cone outer segments in both models. Early and repeated administration of exogenous 11-cis-retinal to Rpe $65^{-1-}$ Rho ${ }^{-1-}$ double knock-out pups prevented these mislocalizations. These results are consistent with a key role of 11-cis-retinal bound to cone opsins for retinal protein sorting, transport, and targeting and, ultimately, cell survival.

\section{Materials and Methods}

Mouse lines. Lrat ${ }^{-1-}$ mice were generated and genotyped as described previously (Batten et al., 2004). Rpe65 ${ }^{-1-}$ mice (Redmond et al., 1998a) were provided by T. Michael Redmond (Laboratory of Retinal Cell and Molecular Biology, National Eye Institute). RPE $65^{-1-} R h o^{-1-}$ mice were the generous gift from Mathias Seeliger (University of Tübingen, Tübingen, Germany). Unless otherwise noted, all experiments were conducted on cyclic light-reared, age-matched animals. Animal experiments were performed in accordance with the policy on the Use of Animals in Neuroscience Research and conformed to the recommendations of the Association of Research for Vision and Ophthalmology. The procedures were approved by the Medical University of South Carolina Animal Care and Use Committee and the Institutional Animal Care and Use Committee at the University of Utah.

11-cis-Retinal injections. rrat $^{-1-}$ and Rpe $65^{-1-}$ Rho ${ }^{-1-}$ mice were housed in the dark starting at day P10. Experimental animals were injected intraperitoneally with 11 -cis-retinal $(0.5 \mathrm{mg}$ per dose) in $100 \mu \mathrm{l}$ of vehicle $(1.0 \%$ ethanol, $10 \%$ bovine serum albumin, and $0.9 \% \mathrm{NaCl})$ on days P10, P13, and P17. Mice were killed at age P20 for the cone antigen localization experiments. Littermate control mice were injected on the same schedule with vehicle alone.

PNA labeling in flat-mounted retinas. Eyes were isolated and placed in PBS, pH 7.4. After removing the cornea, the RPE-choroid layer was separated from the retina-lens complex, which was fixed in $4 \%$ formaldehyde solution in PBS for $4 \mathrm{~h}$ at $4^{\circ} \mathrm{C}$. After washing with PBS (three times for $30 \mathrm{~min}$ each at $4^{\circ} \mathrm{C}$ ), the retinas were incubated with the 0.2 $\mathrm{mg} / \mathrm{ml}$ FITC-conjugated peanut agglutinin (PNA; lectin Arachi hypogaea; Sigma, St. Louis, MO) overnight at $4^{\circ} \mathrm{C}$. Retinas were washed with PBS (three times for $20 \mathrm{~min}$ each at $4^{\circ} \mathrm{C}$ ), mounted on a slide and coverslipped after application of an anti-fade solution (Prolong; Invitrogen, Eugene, OR). The samples were viewed by fluorescence microscopy (Axioplan II; Zeiss, Jena, Germany) using a $100 \mathrm{~W}$ mercury light source and FITC filters. Images were captured and processed (AxioVision soft- ware version 4.5; Diagnostic Instruments, Sterling Heights, MI). Cones were counted from five fields (far dorsal, dorsal, central, ventral, and far ventral) per flat-mounted retina and averaged for six mice of each genotype. Data are expressed as the mean number of cones $/ \mathrm{mm}^{2} \pm \mathrm{SEM}$. For overall changes in cell numbers between the two retinoid cycle mutants and wild-type (WT) retinas, data were analyzed by repeated-measures ANOVA followed by Dunnett's post hoc test $(p<0.05)$; for analysis of regional differences in cone cell counts, one-way ANOVA followed by pairwise Tukey's post hoc test was used $(p<0.05)$.

Immunolocalization by confocal microscopy. Age-matched mouse eyes were immersion fixed for $2 \mathrm{~h}$ using freshly prepared $4 \%$ paraformaldehyde in $0.1 \mathrm{~m}$ phosphate buffer, $\mathrm{pH} 7.4$, and cryoprotected. Eyes were coembedded, frozen, and sectioned at $12 \mu \mathrm{m}$ thickness before incubations for immunocytochemistry as described by Bhosale et al. (2004) and Baehr et al. (2007). Affinity-purified primary antibodies (15-25 $\mu \mathrm{l}$ ) were applied to each group of two to four sections in a humidified, rotating chamber overnight at $4^{\circ} \mathrm{C}$. Propidium iodide (1:3000 dilution; Invitrogen) was added to the solution containing FITC-conjugated secondary antibody. The sections were viewed using a Zeiss LSM 510 inverted laserscanning confocal microscope with a $40 \times 1.3$ numerical aperture oil objective lens and optical slit setting of $<0.9 \mu \mathrm{m}$. Antibodies and dilutions used in this study were described previously (Baehr et al., 2007).

Immunoblotting. Wild-type and $\mathrm{Lrat}^{-1-}$ retinas (one each) at age of P15-P16 were homogenized by sonication. Equal amounts (1/15 of total amount) of the proteins from each retina were loaded on a $12.5 \%$ SDSPAGE gel. After separation, the proteins were transferred to a nitrocellulose filter and probed with primary antibodies as described previously (Zhang et al., 2007). The signals were visualized by ECL (NEN/PerkinElmer, Waltham MA).

Quantitative reverse transcription- $P C R$. Semiquantitative real-time reverse transcription (RT)-PCR was performed using total RNA from WT, $\mathrm{Lrat}^{-1-}$, and Rpe65 $5^{-1-}$ retinas. Total RNAs were extracted from P15 retina, and real-time RT-PCR was performed with $25 \mathrm{ng}$ of RNA as described previously (Baehr et al., 2007). Primers [Gnat2 forward, 5' atgacctgtgctacagacacacagaac; Gnat2 reverse, 5'-gcatgaagcctcagattctaagcttgc; phosphodiesterase 6c (Pde6c) forward, 5'-cagcgccgaagacaaaaagtccaaaac, $P d e 6 c$ reverse, $5^{\prime}$-ttctgtcacttcttggtcactggacag; Gapdh forward, 5'-acccttcattgacctcaactacatgg; Gapdh reverse, 5' atttgatgttagtggggtctcgctcct] amplified $\sim 150 \mathrm{bp}$ fragments. Results were normalized to Gapdh signals for each sample (Baehr et al., 2007).

\section{Results}

\section{Lrat $^{-/-}$cone photoreceptors degenerate rapidly}

In 1-month-old $\mathrm{Lrat}^{-/-}$retinas, cones were unevenly distributed with more cones surviving dorsally than in the ventral and central retina (Fig. $1 A$ ). A similar distribution pattern was observed in Rpe65 ${ }^{-1-}$ mice (Znoiko et al., 2005). Using a repeated-measures ANOVA, cone cell numbers across all five regions were found to vary with genotype, an effect that was region specific (genotype $\times$ region, $\left.F_{(8,16)}=18.9 ; p<0.0001\right)$. Dunnett's post hoc test $(p<$ $0.05)$ confirmed that compared with retinas of WT mice, the total cone population in Rpe $65^{-1-}$ and $\mathrm{Lrat}^{-1-}$ mice was significantly reduced; however, the overall cone population did not differ between the Rpe $65^{-1-}$ and $\mathrm{Lrat}^{-1-}$ retinas. To determine whether cone densities in individual retina areas differ between $\mathrm{Lrat}^{-1-}$, Rpe $65^{-1-}$, and control mice, cell counts were analyzed by oneway ANOVA followed by a pairwise Tukey's post hoc analysis $(p<0.05)$ (Fig. $1 B$ ). The one-way ANOVA across the three genotypes revealed that all regions but the far-dorsal retina varied with genotype $(p<0.0001)$. Post hoc analysis in the remaining four retina areas showed regional differences in cone cell numbers in the central, ventral, and far-ventral retina between $\mathrm{Lrat}^{-1-}$ and Rpe65 ${ }^{-1-}$ mice, but not in the dorsal retina; all pairwise comparisons between $\mathrm{Lrat}^{-1-}$ and control and Rpe $65^{-1-}$ and control mice were statistically significant. We observed a similar dorsal-ventral pattern in dark-reared animals, 

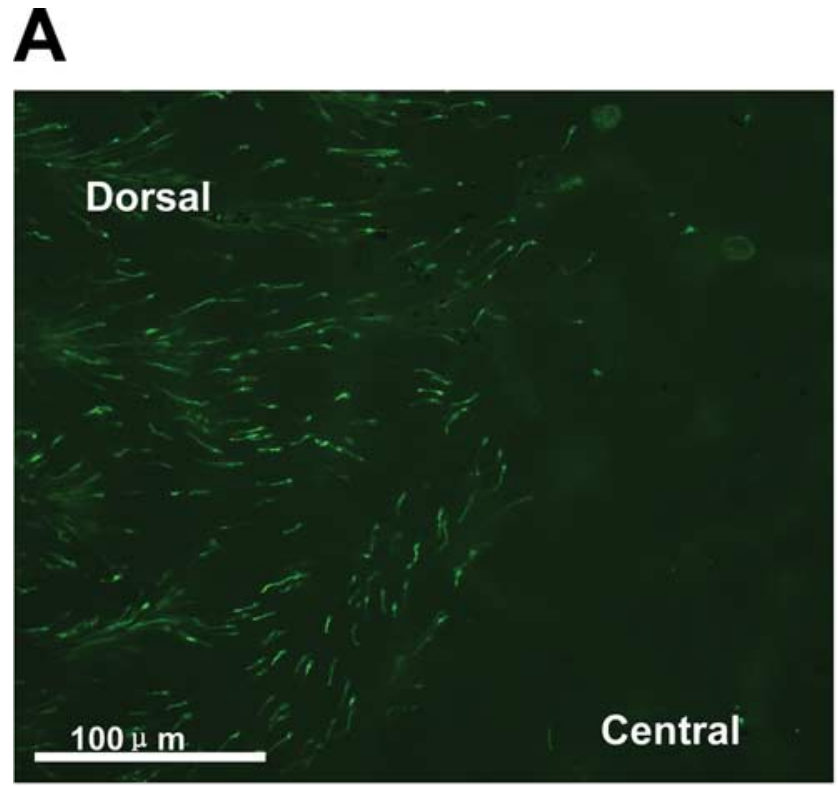

\section{B}

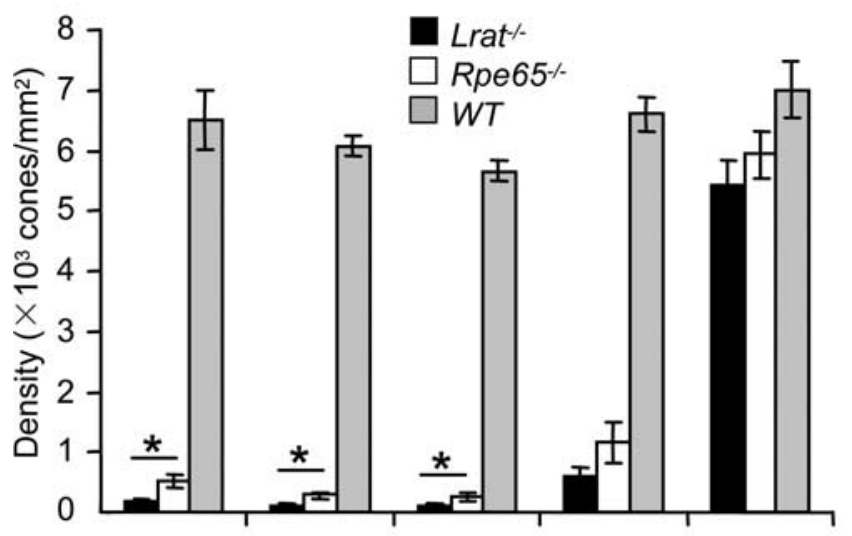

Far Ventral Ventral Central Dorsal Far Dorsal

Figure 1. Cone distribution in $\mathrm{Lrat}^{-1-}$ and Rpe65 $5^{-1-}$ retinas. A, Flat mount. Lrat ${ }^{-1-}$ cones were visualized in mice at 4 weeks of age with FITC-conjugated peanut agglutinin (green) binding to the extracellular cone sheaths. Magnification, 20×. B, Bar graph depicting cone density. Cones were counted from five fields per flat-mounted retina, averaged in six mice from each group. Data are expressed as the mean number of cones $/ \mathrm{mm}^{2} \pm \mathrm{SEM}$. Overall cone cell counts did not differ between the two retinoid cycle mutants based on repeated-measures ANOVA followed by Dunnett's post hoc test $(p<0.05)$, whereas regional differences could be identified using one-way ANOVA followed by pairwise comparison using Tukey's post hoc test $\left({ }^{*} p<0.05\right)$. White bars, Rpe65 ${ }^{-1-}$ mice; black bars, Lrat ${ }^{-1-}$ mice; gray bars, WT mice.

thereby ruling out light damage as the cause of rapid central progression of cone loss.

\section{Mistargeting of cone pigments}

Shortly after eye opening, COSs are well established in P15 WT mice, as evidenced by anti-M/L-opsin labeling (Fig. $2 A$ ). In the P15 $\mathrm{Lrat}^{-1-}$ mouse, M/L-opsin was seen partially mislocalized, with some M/L-opsin present in the short COSs, the somata, and synaptic pedicles (Fig. 2B, arrows), quite similar to the P15 Rpe $65^{-1-}$ mouse (Fig. 2C). Mislocalization and mistrafficking of both S-opsin and M/L-opsin have been shown previously to occur in the Rpe $65^{-1-}$ and the Rpe65 ${ }^{-1-}$ Rho ${ }^{-1-}$ mouse (Rohrer et al., 2005). At P15-P20, COSs were still present in the rrat $^{-1-}$ mouse based on weak immunolabeling for M/L-opsin, but at
P30, cone degeneration and COS disintegration appeared to be far advanced in both models (Fan et al., 2008).

Mistargeting of PMPs, GC1, and R9AP in $\mathrm{Lrat}^{-1-}$ cones

We observed a similar cone pigment mislocalization in a mouse lacking GC1 (Gucy2e), an enzyme responsible for cGMP synthesis in rods and cones (Baehr et al., 2007). This knock-out mouse, a model for recessive cone dystrophy, presented with defects in transport of PMPs essential for cone phototransduction. We therefore investigated the presence of PMPs in the $\mathrm{Lrat}^{-1-}$ COSs and found, by analogy with the Gucy $2 e^{-1-}$ mouse, that acylated and prenylated proteins of the cone phototransduction cascade were diminished (Fig. 3). PDE6 $\alpha^{\prime}$, the catalytic subunit of cone PDE6, was undetectable in COSs by immunocytochemistry at predegenerate P15 (Fig. 3B), whereas WT COSs showed robust presence of this polypeptide (Fig. $3 A$ ). Similarly, both acylated cone T $\alpha$ and farnesylated cone T $\gamma$, strongly present in P15 WT COSs (Fig. 3C,E), were essentially absent in residual mutant COSs (Fig. 3D, F). Faint immunolabeling of apparently downregulated polypeptides was observed in cone inner segments (CISs) and COSs (Fig. 3 D, F, arrows). In contrast, cone arrestin, a soluble protein distributed throughout the cell, was unaffected in the $\mathrm{Lrat}^{-1-}$ cones (supplemental Fig. S3A, available at www. jneurosci.org as supplemental material). In P30 mutant cones, cone PDE6 $\alpha^{\prime}$ (supplemental Fig. S4, available at www. jneurosci.org as supplemental material) and cone $\mathrm{T} \alpha$ (data not shown) were undetectable by immunocytochemistry. Absence of these signal-amplifying polypeptides is predicted to attenuate cone phototransduction severely.

GRK1 (opsin kinase) levels are very high in P15 WT COSs, as indicated by the intense yellow fluorescence generated by colabeling with anti-mouse cone arrestin (mCAR) (Fig. 3I, arrows) and the strongly labeled COSs [supplemental Fig. S2 (arrows), available at www.jneurosci.org as supplemental material]. In contrast, GRK1 was much diminished in P15 $\mathrm{Lrat}^{-1-}$ COSs (Fig. $3 J)$. A farnesylated protein associated with the synaptic ribbon, complexin III (CPX III) (Reim et al., 2005), trafficked normally to the cone pedicles (Fig. 3G,H), suggesting that mistrafficking in $\mathrm{Lrat}^{-1-}$ cones may be specific for PMPs associated with the COSs.

GC1, the sole particulate guanylate cyclase of cones, is expressed at high levels and accumulates predominantly in WT COSs, as revealed by colabeling with mCAR (Fig. $3 K$ ). At P15, GC1 levels were apparently much reduced in $\mathrm{Lrat}^{-1-}$ COSs (Fig. $3 L)$. Another transmembrane protein expressed at high levels in cones, R9AP, the membrane anchor of the GTPase-activating complex RGS9/G $\beta 5$, also is much reduced in mutant COSs (Fig. $3 M, N)$. Severely reduced levels of GRK1, GC1, and R9AP in $\mathrm{Lrat}^{-1-}$ COSs are predicted to affect phosphorylation of cone pigments and cone phototransduction.

\section{Membrane-associated cone phototransduction protein levels are reduced}

By immunoblot, cone-specific PDE6 $\alpha^{\prime}$ and cone T $\alpha$ polypeptides were less abundant in P15 $\mathrm{Lrat}^{-1-}$ than in age-matched WT retinas (Fig. 4A). GRK1, abundantly present in rod outer segments (ROSs), which degenerate very slowly (Batten et al., 2005), and the rod-specific PDE6 $\alpha$ subunit were unaffected. Lower levels of cone T $\alpha$ and cone PDE $\alpha^{\prime}$ could be caused by effects on gene regulation or posttranscriptional/posttranslational events. We therefore measured P15 Gnat 2 and Pde6c mRNA levels relative to those of the housekeeping Gapdh mRNA to probe for changes in gene regulation (Fig. $4 B$ ). Quantitative RT-PCR results showed 

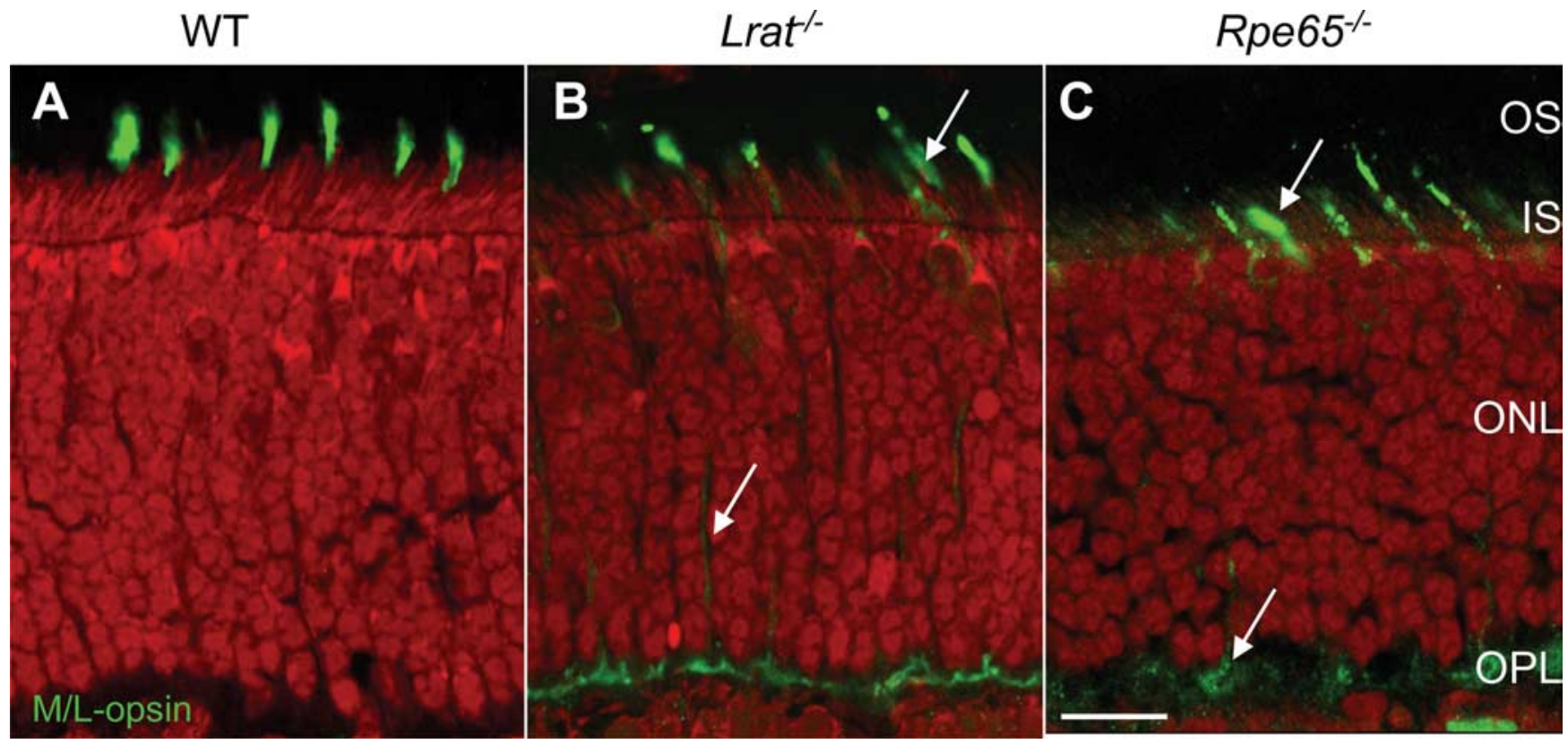

Figure 2. Confocal immunolocalization of $\mathrm{M} / \mathrm{L}-\mathrm{opsin}$ in $\mathrm{WT}, \mathrm{Lrat}^{-1-}$, and Rpe65 ${ }^{-/-}$cones at postnatal day $15 . \mathrm{A}-\mathrm{C}, \mathrm{M} / \mathrm{L}-\mathrm{opsin}$ in WT and mutant retinas at P15 $\pm 1 \mathrm{~d}$. Note that M/L-opsin mislocalizes in part to the inner segment, perinuclear region, and synaptic terminal of mutant cones ( $\boldsymbol{B}, \boldsymbol{C}$, arrows). Immunoreactivity for M/L-opsin in the mutant retinas is mislocalized early (P15) and nearly absent at P30 (data not shown). All retina sections passed through the optic nerve, and in each case, photoreceptors were imaged ventral (inferior) to the nerve where the degeneration was most advanced. Nuclei are contrasted with propidium iodide (red). Scale bar, $10 \mu \mathrm{m}$. OS, Outer segments; IS, inner segments; ONL, outer nuclear layer; OPL, outer plexiform layer. Scale bars and abbreviations are the same for Figures 2, 3, and 5 and supplemental Figures S1-S3 (available at www.jneurosci.org as supplemental material).

that levels of Gnat2 and Pde6c mRNAs in P15 WT, Lrat $^{-1-}$, and $R$ pe $65^{-1-}$ retinas were similar, suggesting that reduction of cone $\mathrm{T} \alpha$ and $\mathrm{PDE} 6 \alpha^{\prime}$ protein levels occurs posttranslationally.

\section{1-cis-Retinal is essential for normal cone opsin trafficking}

In the Rpe $65^{-1-}$ retina, cone opsins and peripheral membrane proteins mislocalize in a manner similar to that observed in Lrat $^{-1-}$ photoreceptors (Fig. 5). S-opsin was faintly detectable only in the inner segments at P15 (Fig. 5B), whereas M/L-opsin was, in part, apparently transported to the COSs (Fig. $5 F$ ). Thus, both $\mathrm{Lrat}^{-1-}$ and Rpe65 $5^{-1-}$ cones possess shortened COSs that can be labeled by antibodies directed against M/L-opsin (Fig. $5 F$ ) and cone arrestin (Fig. $3 \mathrm{~J}, L$ ), as well as cone sheaths that are positive for PNA-lectin (supplemental Fig. S1, available at www. jneurosci.org as supplemental material). PDE $6 \alpha^{\prime}$, cone T $\alpha$, and GC1 labeling in Rpe65 $5^{-1-}$ retina (Fig. 5J,N,R) was nearly identical to that seen in $\mathrm{Lrat}^{-1-}$ mice (Fig. $3 \mathrm{~B}, \mathrm{D}, \mathrm{L}$ ). After administration of 11-cis-retinal to Rpe65 $5^{-l-}$ Rho ${ }^{-1-}$ pups (on postnatal days 10, 13, and 17), both opsins were seen in the COSs at P20 (Fig. $5 D, H$ ), as observed at P25 in a previous study (Rohrer et al., 2005). S-opsin appeared to be localized normally, whereas the effect on M/L-opsin localization was corrected only partially (Fig. $5 D, H)$. Relative to untreated mutant mice, more COSs were labeled after 11-cis-retinal treatment, indicating improved opsin migration into the COSs. 11-cis-retinal treatment in the $\mathrm{Lrat}^{-1-}$ mouse on $\mathrm{Rho}^{+/+}$background produced less pronounced results because of the large pool of rod opsin competing for the injected 11-cis-retinal (data not shown). These results establish that 11-cis-retinal is essential for normal cone opsin trafficking during postnatal cone development.

Administration of 11-cis-retinal corrects abnormal trafficking of peripheral membrane proteins in Rpe $65^{-/-}$mutant mice PDE6 $\alpha^{\prime}$ and cone T $\alpha$ were essentially undetectable in COSs of P15 rrat $^{-1-}$ (Fig. 3 B,D) and Rpe65 ${ }^{-I-}$ (Fig. 5J,N) retinas. Only weak punctuate labeling could be detected in $\mathrm{P} 15$ Rpe65 ${ }^{-1-}$ (Fig. $5 \mathrm{~J}, \mathrm{~N}$ ) and P20 Rpe65 ${ }^{-/-}$Rho ${ }^{-1-}$ (Fig. $5 \mathrm{~K}, \mathrm{O}$ ) CISs, consistent with failure of these peptides to traffic to the COSs. No mislocalization to the pedicle was observed for these PMPs (data not shown). Differential expression of GC1, an enzyme present in rods and cones, was also examined. As expected, GC1 levels were maintained in WT and mutant ROSs (Fig. 5Q,R), consistent with the slow degeneration of Rpe65 $5^{-1-}$ rods and presence of opsin in the ROSs (Redmond et al., 1998b). However, although anti-GC1 antibody identified COSs readily in P15 WT retina (Fig. 5Q, arrows), the identical antibody solution was unable to detect COSs in P15 Rpe65 $5^{-l-}$ (Fig. 5R) or P20 Rpe65 $5^{-l-}$ Rho ${ }^{-l-}$ retinas (Fig. $5 S)$. This result was interpreted to suggest that GC1 had not transported to the mutants' COSs. As with restoration of cone pigment distributions to the cone outer segments (Fig. 5D, $H$ ), administration of exogenous 11-cis-retinal resulted in normal trafficking of PDE6 $\alpha^{\prime}$ (Fig. $5 L$ ), cone T $\alpha$ (Fig. 5P), and GC1 (Fig. 5T) to the COSs.

\section{Discussion}

The main observations of our study of $\mathrm{Lrat}^{-1-}$ and Rpe65 cone photoreceptors are as follows: (1) mutant cones develop and form short COSs, but degenerate rapidly in the ventral (inferior) and central retina regions; (2) cone transmembrane proteins ( $\mathrm{S}$ opsin, M/L-opsin, GC1, and R9AP) and peripherally membraneassociated proteins (T $\alpha, \operatorname{PDE} 6 \alpha^{\prime}$, and GRK1) fail to traffic properly to the outer segment; (3) cone T $\alpha$ and PDE6 $\alpha^{\prime}$ protein levels are decreased as a result of posttranslational degradation; and (4) early treatment with 11-cis-retinal promotes correct targeting of opsins and membrane-associated phototransduction components to the cone outer segments of Rpe $65^{-1-} \mathrm{Rho}^{-1-}$ double knock-out mice.

Cones of both $\mathrm{Lrat}^{-1-}$ and Rpe65 ${ }^{-1-}$ mutants degenerate rapidly after eye opening (Fig. 1), although cone losses sectorially reveal significant differences in the rate of degeneration. Cone 
degeneration proceeds rapidly in the central retina, but is delayed dorsally (Znoiko et al., 2005); cones survive much longer in far-dorsal regions. Incomplete cone loss and residual cone function were also observed in RPE65-LCA patients (Jacobson et al., 2007). Mechanisms leading to increased sensitivity of central cones to RPE65 loss are currently not understood. In human, the central RPE layer shows a fourfold higher retinoid isomerase activity than the more peripheral RPE, suggesting that robust RPE65-based visual chromophore production is important for macular cones (Jacobson et al., 2007). Additional processes may exist that generate 11-cis-retinal, supplementing the major RPE65-dependent pathway in far-dorsal cones (Mata et al., 2005; Muniz et al., 2007; Schonthaler et al., 2007).

Immunohistochemistry indicates that cone opsins distribute throughout the mutant cone photoreceptors with only minor targeting to the COSs (Fig. 2). Failure to traffic properly appears to be a post-Golgi targeting disorder, because opsins are exported from the endoplasmic reticulum (ER) but apparently do not reach the outer segment. The congruence of cone opsin mislocalizations in $\mathrm{Lrat}^{-1-}$ and Rpe65 ${ }^{-1-}$ mice suggests that these targeted deletions converge at a common degenerative pathway, e.g., protein sorting during vesicular transport. Using a variety of doublelabeling techniques, we found that GC1 was severely reduced in mutant COSs (Figs. 3, 5) suggesting that the cone pigments, GC1, and R9AP, which differ in abundance by several orders of magnitude (Liu et al., 2007), may be linked in a common transport pathway. In addition to the mislocalization of cone pigments and GC1 in $\mathrm{Lrat}^{-1-}$ and Rpe65 ${ }^{-1-}$ cones, we were unable to detect the peripherally membrane-associated proteins (cone $\mathrm{T} \alpha$, cone T $\gamma, \mathrm{PDE} 6 \alpha^{\prime}$, and GRK1) in the mutant COSs (Fig. 5). Common features of these proteins are posttranslational prenylation and acylation, and association with the ER for processing (Zhang et al., 2007). It is likely that these proteins also require vesicular transport to the outer segments. For GRK1 and cone PDE6 $\alpha^{\prime}$, but not rod and cone transducins, this transport is mediated by the prenyl binding protein, $\operatorname{PrBP} / \delta$ (Zhang et al., 2007). Absence of these nondiffusible PMPs in Rpe65 ${ }^{-1-}$ and $\mathrm{Lrat}^{-1-}$ COSs suggests that their transport also depends on the presence of 11-cis-retinal and cone pigment formation.

As abundant plasma membrane structural proteins, cone pigments presumably

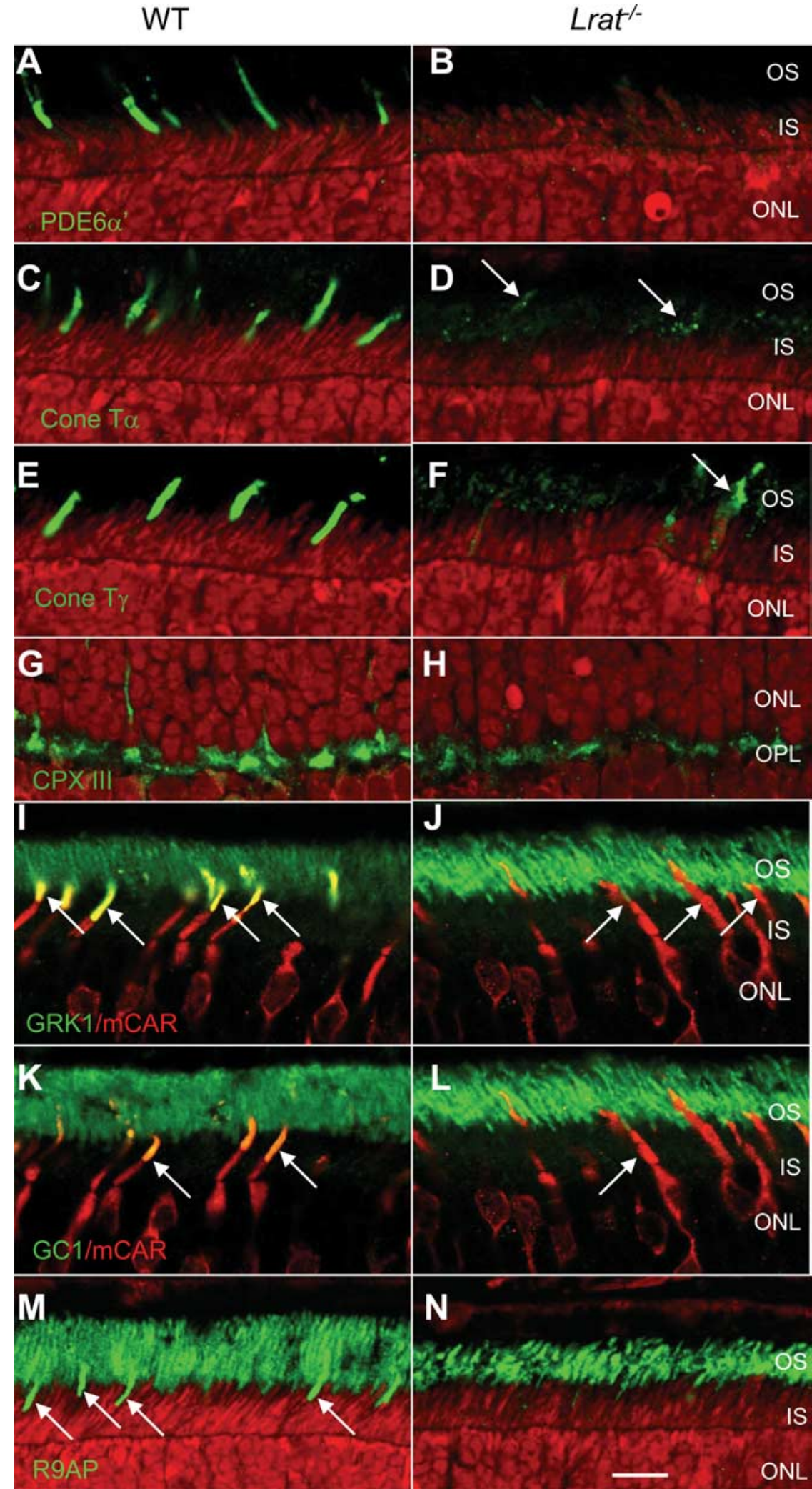

Figure 3. Immunolocalization of peripheral membrane-associated proteins, GC1, and R9AP in P15 WT and $\mathrm{Lrat}^{-1-}$ retinas. P15 littermate WT and Lrat $^{-1-}$ eyes were coembedded, and central retina sections were probed simultaneously. Sections of P15 WT and $\mathrm{Lrat}^{-1-}$ retinas were probed using anti-PDE6 $\alpha^{\prime}(\boldsymbol{A}, \boldsymbol{B})$, anti-cone $\mathrm{T} \alpha(\boldsymbol{C}, \boldsymbol{D})$, anti-cone $\mathrm{T} \gamma(\boldsymbol{E}, \boldsymbol{F})$, anti-CPX III $(\boldsymbol{G}, \boldsymbol{H})$, anti-GRK1 $(\boldsymbol{I}, \boldsymbol{J})$, anti-GC1 $(\boldsymbol{K}, \boldsymbol{L})$, and anti-R9AP $(\boldsymbol{M}, \boldsymbol{N})$ antibodies. Bright red nuclei in $\boldsymbol{B}$ and $\boldsymbol{H}$ indicate apoptotic photoreceptors. $I-L$, Central retina sections were probed simultaneously with either monoclonal anti-GRK1 antibody $(I, J$, green) or anti-GC1 antibody ( $K, \boldsymbol{L}$, green) and polyclonal anti-cone arrestin antibody to identify cones $(\boldsymbol{I}-\boldsymbol{L}$, red). Colocalization of mCAR with GRK1 (I, yellow at arrows) or GC1 (K, arrows) is evident in WT COSs, whereas in Lrat $^{-1-}$ sections, COSs are largely devoid of GRK1 $(\boldsymbol{J})$ and GC1 (L). 
A

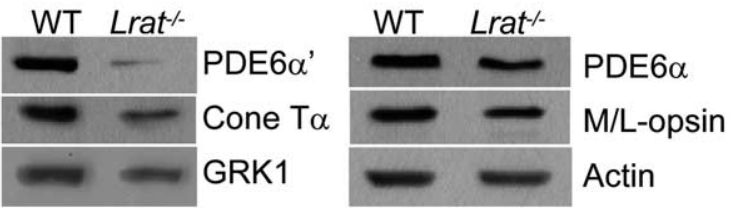

B

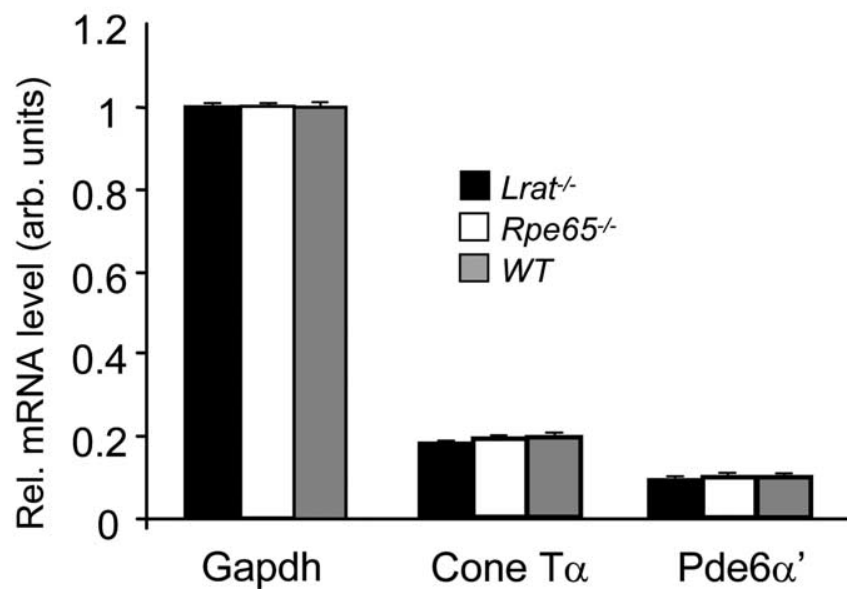

Figure 4. Immunoblot and real-time PCR. $A$, Summary immunoblot data from P15 WT and $\mathrm{Lrat}^{-1-}$ retina lysates. Blots were probed with antibodies directed against cone PDE $6 \alpha^{\prime}$, cone T $\alpha$, GRK1, PDE $6 \alpha$, or M/L-opsin. Internal loading controls with $\beta$-actin were included for each immunoblot. The results shown are representative of several blots using different animals of identical genotypes. Downregulation of cone phototransduction polypeptides is apparent in retina extracts of $P 15 \mathrm{Lrat}^{-1-}$ mice. $B$, Quantitative real-time $P C R$ to determine relative transcript levels of Gnat2 and Pde6c. RNA samples of P15 rrat $^{-/-}$, RPE65-/-, and wild-type retinas were reverse transcribed and amplified using Gnat2-, Pde6c-, and Gapdh-specific primers. Relative mRNA levels, normalized to the fluorescence of the Gapdh standard, are shown as bar graphs. Experiments for each gene were done in parallel and repeated three times.

contribute to COS stability, as does rhodopsin to rod outer segment formation and stability (Humphries et al., 1997; Lem et al., 1999). The transmembrane protein GC1 occurs in the COSs at much lower abundance with an unknown contribution to membrane stability. Therefore, $\mathrm{Lrat}^{-1-}$ and Rpe65 ${ }^{-1-}$ cone degeneration may be attributed, in large part, to the severe reduction of cone opsins in the outer segment membrane causing destabilization. The pattern of cone opsin mislocalization observed in $\mathrm{Lrat}^{-1-}$ and $\mathrm{Rpe} 65^{-1-}$ mice resembles those reported in retinas

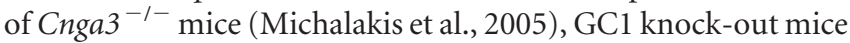
(Baehr et al., 2007; Karan et al., 2007), and, to some extent, CFH (complement factor H) knock-out animals (Coffey et al., 2007). Common to all, cone outer segments are unstable and cone photoreceptors degenerate.

Cone opsin mislocalization can be corrected, and degeneration arrested, by repeated administration of 11-cis-retinal at an early age (Fig. 5D,H,L,P,T). The 11-cis-retinal experiments involved Rpe65 $5^{-1-}$ mice with a $R h o^{-l-}$ background to maximize this therapeutic effect, by minimizing the competition by rod opsin for the 11-cis-retinal, because rods are known to compete favorably with cones for limited amounts of chromophore (Matsumoto et al., 1975; Shichida et al., 1990). Chromophore linkage to the cone opsins is known to be weaker, especially to $\mathrm{M} / \mathrm{L}$ opsin, implying that cone opsins may possess more accessible binding sites (Kefalov et al., 2005). Our data suggest that 11-cisretinal binding to cone opsin is required for correct targeting of pigment. In the absence of appropriate cone opsin trafficking, many COS-associated proteins also do not traffic properly to the COSs. One explanation might be that 11-cis-retinal induces a conformational change in cone opsins that is required for recognition by a chaperone or for formation of a vesicular transport carrier that will permit other COS proteins to be cotransported and targeted correctly.

\section{References}

Baehr W, Karan S, Maeda T, Luo DG, Li S, Bronson JD, Watt CB, Yau K-W, Frederick JM, Palczewski K (2007) The function of guanylate cyclase 1 (GC1) and guanylate cyclase 2 (GC2) in rod and cone photoreceptors. J Biol Chem 282:8837-8847.

Batten ML, Imanishi Y, Maeda T, Tu DC, Moise AR, Bronson D, Possin D, Van Gelder RN, Baehr W, Palczewski K (2004) Lecithin-retinol acyltransferase is essential for accumulation of all-trans-retinyl esters in the eye and in the liver. J Biol Chem 279:10422-10432.

Batten ML, Imanishi Y, Tu DC, Doan T, Zhu L, Pang J, Glushakova L, Moise AR, Baehr W, Gelder RN, Hauswirth WW, Rieke F, Palczewski K (2005) Pharmacological and rAAV gene therapy rescue of visual functions in a blind mouse model of Leber congenital amaurosis. PLoS Med 2:e333.

Bhosale P, Larson AJ, Frederick JM, Southwick K, Thulin CD, Bernstein PS (2004) Identification and characterization of a Pi isoform of glutathione S-transferase (GSTP1) as a zeaxanthin-binding protein in the macula of the human eye. J Biol Chem 279:49447-49454.

Coffey PJ, Gias C, McDermott CJ, Lundh P, Pickering MC, Sethi C, Bird A, Fitzke FW, Maass A, Chen LL, Holder GE, Luthert PJ, Salt TE, Moss SE, Greenwood J (2007) Complement factor H deficiency in aged mice causes retinal abnormalities and visual dysfunction. Proc Natl Acad Sci USA 104:16651-16656.

Ebrey T, Koutalos Y (2001) Vertebrate photoreceptors. Prog Retin Eye Res 20:49-94.

Fan J, Rohrer B, Frederick JM, Baehr W, Crouch RK (2008) Rpe65-/- and Lrat - / - mice: comparable models of Leber congenital amaurosis. Invest Ophthalmol Vis Sci, in press.

Humphries MM, Rancourt D, Farrar GJ, Kenna P, Hazel M, Bush RA, Sieving PA, Sheils DM, McNally N, Creighton P, Erven A, Boros A, Gulya K, Capecchi MR, Humphries P (1997) Retinopathy induced in mice by targeted disruption of the rhodopsin gene. Nat Genet 15:216-219.

Jacobson SG, Aleman TS, Cideciyan AV, Heon E, Golczak M, Beltran WA, Sumaroka A, Schwartz SB, Roman AJ, Windsor EA, Wilson JM, Aguirre GD, Stone EM, Palczewski K (2007) Human cone photoreceptor dependence on RPE65 isomerase. Proc Natl Acad Sci USA 104:15123-15128.

Jin M, Li S, Moghrabi WN, Sun H, Travis GH (2005) Rpe65 is the retinoid isomerase in bovine retinal pigment epithelium. Cell 122:449-459.

Karan S, Zhang H, Li S, Frederick JM, Baehr W (2007) A model for transport of membrane-associated phototransduction polypeptides in rod and cone photoreceptor inner segments. Vision Res 48:442-452.

Kefalov VJ, Estevez ME, Kono M, Goletz PW, Crouch RK, Cornwall MC, Yau KW (2005) Breaking the covalent bond-a pigment property that contributes to desensitization in cones. Neuron 46:879-890.

Lem J, Krasnoperova NV, Calvert PD, Kosaras B, Cameron DA, Nicol, Makino CL, Sidman RL (1999) Morphological, physiological, and biochemical changes in rhodopsin knockout mice. Proc Natl Acad Sci USA 96:736-741.

Liu Q, Tan G, Levenkova N, Li T, Pugh Jr EN, Rux JJ, Speicher DW, Pierce EA (2007) The proteome of the mouse photoreceptor sensory cilium complex. Mol Cell Proteomics 6:1299-1317.

Maeda A, Maeda T, Sun W, Zhang H, Baehr W, Palczewski K (2007) Redundant and unique roles of retinol dehydrogenases in the mouse retina. Proc Natl Acad Sci USA 104:19565-19570.

Mata NL, Ruiz A, Radu RA, Bui TV, Travis GH (2005) Chicken retinas contain a retinoid isomerase activity that catalyzes the direct conversion of all-trans-retinol to 11-cis-retinol. Biochemistry 44:11715-11721.

Matsumoto H, Tokunaga F, Yoshizawa T (1975) Accessibility of the iodopsin chromophore. Biochim Biophys Acta 404:300-308.

Michalakis S, Geiger H, Haverkamp S, Hofmann F, Gerstner A, Biel M (2005) Impaired opsin targeting and cone photoreceptor migration in 
the retina of mice lacking the cyclic nucleotidegated channel CNGA3. Invest Ophthalmol Vis Sci 46:1516-1524.

Moiseyev G, Chen Y, Takahashi Y, Wu BX, Ma JX (2005) RPE65 is the isomerohydrolase in the retinoid visual cycle. Proc Natl Acad Sci USA 102:12413-12418.

Muniz A, Villazana-Espinoza ET, Hatch AL, Trevino SG, Allen DM, Tsin AT (2007) A novel cone visual cycle in the cone-dominated retina. Exp Eye Res 85:175-184.

Palczewski K (2006) G protein-coupled receptor rhodopsin. Annu Rev Biochem 75:743-767.

Redmond TM, Yu S, Lee E, Bok D, Hamasaki D, Chen N, Goletz P, Ma JX, Crouch RK, Pfeifer K (1998a) Rpe65 is necessary for production of 11-cis-vitamin A in the retinal visual cycle. Nat Genet 20:344-351.

Redmond TM, Yu S, Lee E, Bok D, Hamasaki D, Pfeifer K (1998b) Characterization of a RPE65 knockout mouse: a model for Leber's congenital amaurosis type II. Invest Ophthalmol Vis Sci 39:S643.

Redmond TM, Poliakov E, Yu S, Tsai JY, Lu Z, Gentleman S (2005) Mutation of key residues of RPE65 abolishes its enzymatic role as isomerohydrolase in the visual cycle. Proc Natl Acad Sci USA 102:13658-13663.

Reim K, Wegmeyer H, Brandstatter JH, Xue M, Rosenmund C, Dresbach T, Hofmann K, Brose N (2005) Structurally and functionally unique complexins at retinal ribbon synapses. J Cell Biol 169:669-680.

Rohrer B, Goletz P, Znoiko S, Ablonczy Z, Ma JX, Redmond TM, Crouch RK (2003) Correlation of regenerable opsin with rod ERG signal in Rpe65-/- mice during development and aging. Invest Ophthalmol Vis Sci 44:310-315.

Rohrer B, Lohr HR, Humphries P, Redmond TM, Seeliger MW, Crouch RK (2005) Cone opsin mislocalization in Rpe65-/- mice: a defect that can be corrected by 11-cis retinal. Invest Ophthalmol Vis Sci 46:3876-3882.

Schonthaler HB, Lampert JM, Isken A, Rinner O, Mader A, Gesemann M, Oberhauser V, Golczak M, Biehlmaier O, Palczewski K, Neuhauss SC, von Lintig J (2007) Evidence for RPE65independent vision in the cone-dominated zebrafish retina. Eur J Neurosci 26:1940-1949.

Shichida Y, Kato T, Sasayama S, Fukada Y, Yoshizawa T (1990) Effects of chloride on chicken iodopsin and the chromophore transfer reactions from iodopsin to scotopsin and B-photopsin. Biochemistry 29:5843-5848.

Van Hooser JP, Liang Y, Maeda T, Kuksa V, Jang GF, He YG, Rieke F, Fong HK, Detwiler PB, Palczewski K (2002) Recovery of visual functions in a mouse model of Leber congenital amaurosis. J Biol Chem 277:19173-19182.

Wald G (1968) The molecular basis of visual excitation. Nature 219:800-807.

Zhang H, Li S, Doan T, Rieke F, Detwiler PB, Frederick JM, Baehr W (2007) Deletion of PrBP/ $\delta$ impedes transport of GRK1 and PDE6 catalytic subunits to photoreceptor outer segments. Proc Natl Acad Sci USA 104:8857-8862.

Znoiko SL, Rohrer B, Lu K, Lohr HR, Crouch RK, Ma JX (2005) Downregulation of conespecific gene expression and degeneration of cone photoreceptors in the Rpe65-/- mouse at early ages. Invest Ophthalmol Vis Sci 46: 1473-1479.

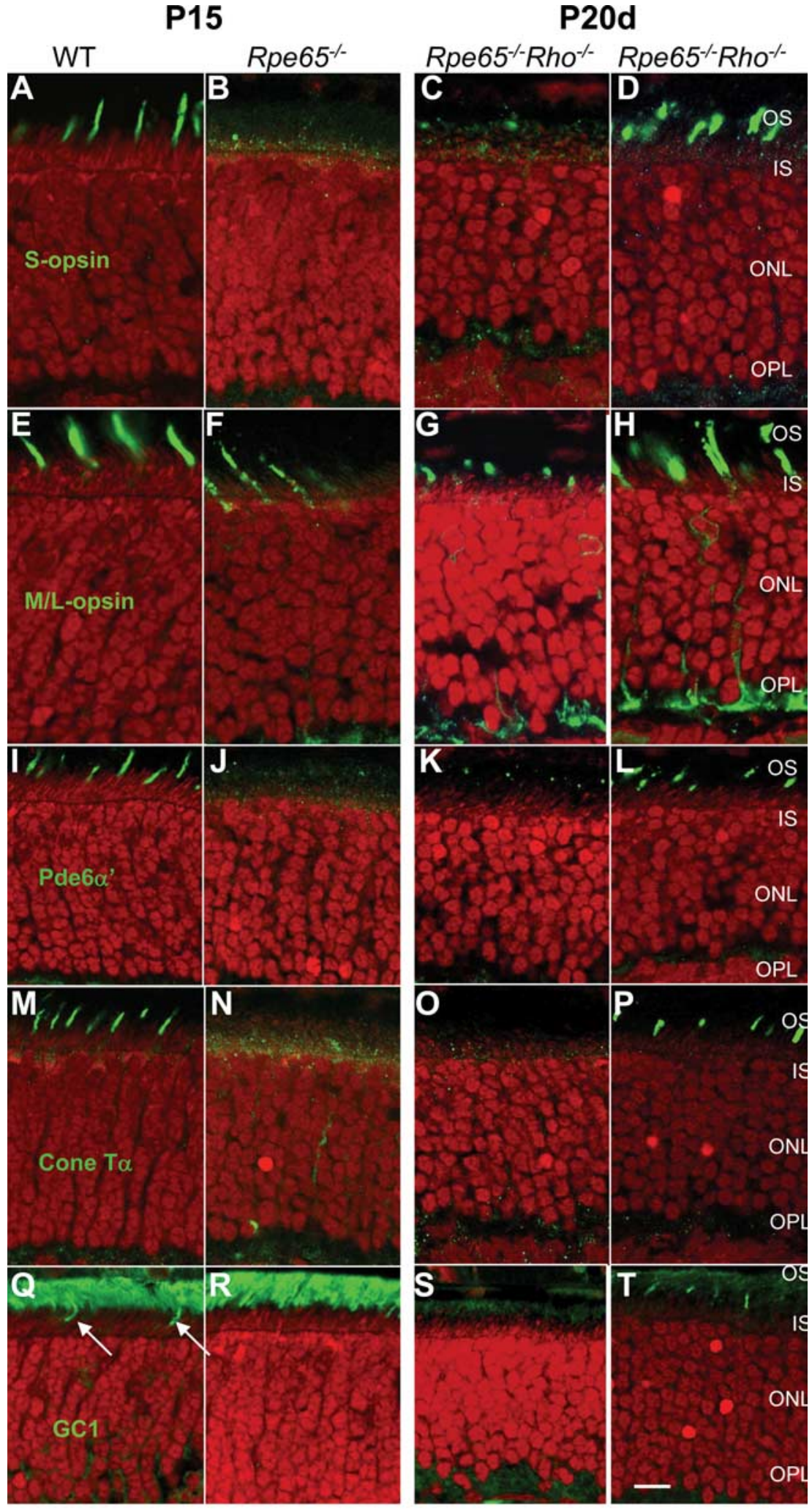

no 11-cis + 11-cis

Figure 5. Immunocytochemistry of $\mathrm{P} 15$ Rpe $65^{-/-}$and $\mathrm{P} 20 \mathrm{~d}$ Rpe $65^{-/-}$Rho ${ }^{-/-}$retina: 11-cis-retinal administration promotes trafficking of cone opsin and peripheral membrane proteins. Left, Immunocytochemistry of WT and Rpe65 ${ }^{-1-}$ cryosections of 15 -d-old retinas. Note that M/L-opsin is mislocalized $(\boldsymbol{F})$, whereas $S$-opsin is barely detectable $(\boldsymbol{B})$ in central, inferior Rpe65 ${ }^{-I-}$ retina. Right, Effect of 11-cis-retinal application on P20 Rpe65 ${ }^{-I-}$ Rho ${ }^{-I-}$ cones. P2Od indicates that mice were kept in the dark after application. Note that in untreated animals, $\operatorname{COS}$ antigens are mislocalized or undetectable, whereas in 11-cis-retinal treated animals, $\operatorname{COS}$ s are elongated and $\operatorname{COS}$ antigens are present. Antibodies tested include the following: anti-Sopsin $(\boldsymbol{A}-\boldsymbol{D})$; anti-M/L-opsin $(\boldsymbol{E}-\boldsymbol{H})$; anti-PDE6 $\alpha^{\prime}(\boldsymbol{I}-\mathbf{L})$; anti-cone T $\alpha(\boldsymbol{M}-\mathbf{P})$; and anti-GC1 (Q- $\left.\boldsymbol{T}\right)$. Nuclei are revealed by propidium iodide (red). 